\title{
Summary of the first annual Genitourinary Medical Oncology Conference Renal Cell Carcinoma Forum
}

\author{
Lori A. Wood, MD, MSc; ${ }^{*}$ Bernard Escudier, MD; ${ }^{\dagger}$ Neil Reaume, $M D^{\ddagger}$
}

\begin{abstract}
The first annual Canadian Genitourinary Medical Oncology Conference was held in June 2006 before the Canadian Urology Association Annual Meeting. This article summarizes 3 presentations that took place as part of the Renal Cell Carcinoma Forum: "Treatment of Metastatic Renal Cell Carcinoma: 2006 and Beyond" was presented by Dr. Bernard Escudier; "Practical Experience with Targeted Therapy," by Dr. Lori Wood; and "Sarcomatoid Renal Cell Carcinoma," by Dr. Neil Reaume.
\end{abstract}

$\mathrm{T}$ he purpose of the first annual Genitourinary Medical Oncology Conference, held in Halifax, Nova Scotia, on June 24, 2006, was to discuss genitourinary oncology topics, in particular renal cell carcinoma (RCC) and testicular cancer; to provide an opportunity for genitourinary medical oncologists to network; to promote medical oncologists' attendance at the Canadian Uro-oncology Group meeting (June 25, 2006) and the Canadian Urological Association meeting (June 25-28, 2006) to provide an opportunity for further interaction with our urology colleagues; and to promote collaboration and interaction between the genitourinary medical oncology and urology disciplines.

Our purpose in this paper is to summarize the 3 presentations given at the Renal Cell Carcinoma Forum.

\section{Treatment of metastatic renal cell carcinoma: 2006 and beyond}

Dr. Bernard Escudier, professor of medicine at the Institut Gustave Roussy in France, presented his talk titled, "Treatment of Metastatic Renal Cell Carcinoma: 2006 and Beyond." He has conducted and participated in a number of studies of RCC with immunotherapy and, more recently, with sorafenib.

Discussion centred on exciting data about the targeted therapies sorafenib, ${ }^{1,2}$ sunitinib ${ }^{3,4}$ and temsirolimus, ${ }^{5}$ reported at the American Society of Clinical Oncology (ASCO) meetings in 2005 and 2006. These data have recently been published in the New England Journal of Medicine in January 2007. 6,7 The presentation was structured in 2 parts: what is known, and what remains to be discovered about these new therapies for patients with RCC.

\section{What is known about RCC}

\section{Interferon improves survival for patients with RCC}

The problem with interferon is that the improvement in survival that it offers for patients with RCC is small. In the Medical Research Council phase 3 trial $^{8}$ that randomized 335 patients to receive either interferon or medroxyprogesterone, those who received interferon had a 8.5-month median survival compared with 6 months for those who received medroxyprogesterone $(p=0.017)$. The improvement in survival, however, may be in only a subset of patients. For example, in the recently reported PERCY Quattro study presented at the ASCO meeting in 2005, ${ }^{9}$ when intermediate-risk patients ( $>1$ metastatic site and Karnofsky Performance Status $\geq 80$ ) were randomized to receive medroxyprogesterone, interferon, interleukin-2 (IL-2) or an interferon and IL-2 combination, the median survival was not statistically different in any of the 4 groups (range 14.9-16.8 mo).

\section{IL-2 and interferon give higher response rates}

In the CRECY trial, ${ }^{10}$ in which 425 patients were randomized to receive IL-2, interferon, or a combination, the combination of IL-2 and interferon had the highest response rates $(18.6 \%)$. The response rate for IL-2 alone was $6.5 \%$ and for interferon alone, $7.5 \%$. However, there was no proven overall survival benefit of this combination therapy. Nor are there studies demonstrating improvement in survival with high-dose intravenous IL-2. However, it is the only registered drug in the United States. Moreover, interferon has been accepted as the standard treatment in most randomized studies, even by the Food and Drug Administration. 
The probability of response to immunotherapy is likely dependent on prognostic factors

Response to immunotherapy is likely dependent on recognized and still unrecognized prognostic factors. For example, if a patient has more than 1 metastatic site, rather than only 1 site, the chance of the tumour responding to immunotherapy is less. Other important prognostic factors may be liver metastases and mediastinal involvement at the time of diagnosis. Likely, immunotherapy has a real impact on only about $20 \%$ of patients. That being said, for those $20 \%$, the benefit may be quite real and significant.

\section{$R C C$ is a capricious disease}

All physicians and surgeons who treat metastatic kidney cancer have had patients who had spontaneous regressions or stable disease after observation alone. Therefore, for some patients, it is important to simply watch them.

\section{RCC has biological differences that are logical to target}

The biological and molecular differences of RCC, such as the von Hippel-Lindau protein, hypoxiainducible factor, vascular endothelial growth factor (VEGF) and platelet-derived growth factor, make logical targets for therapy.

What is known about the effect of VEGF tyrosine-kinase inhibitors on RCC?

\section{VEGF tyrosine-kinase inhibitors shrink tumours}

Impressive radiographic changes can be seen when RCC is treated with VEGF tyrosine-kinase inhibitors. ${ }^{1,2}$

\section{VEGF tyrosine-kinase inhibitors decrease tumour vascularization}

Decreased tumour vascularization has been observed on CT scan imaging and on dynamic contrast-enhanced Doppler ultrasound (DCE-US). In their abstract, presented at the European Cancer Conference (ECCO) 2005 meeting, Dr. Lamuraglia and colleagues ${ }^{11}$ reported on the results of their study of 30 patients ( 9 on sorafenib and 21 on placebo) with CT scan imaging and DCE-US. Combining morphological and dynamic parameters, they defined a good response as a decrease in contrast uptake exceeding $10 \%$ and stable or decreased tumour volume. They found some correlation between the DCE-US findings and progression-free survival. More research is needed in this field.

\section{VEGF tyrosine-kinase inhibitors improve progression-free survival}

Data from the second-line TARGET study ${ }^{1,2}$ showed progression-free survival for patients on sorafenib doubled that for patients on placebo (5.5 mo v. $2.8 \mathrm{mo} ; p<0.01)$. Data from the first-line study ${ }^{4}$ of sunitinib and interferon also showed an improvement in progression-free survival for patients on sunitinib compared with that for patients on interferon (11 mo v. 5 mo; $p<0.001$ ).

\section{VEGF tyrosine-kinase inhibitors are less toxic}

The incidence of grade 3 or 4 treatment-related adverse events in the TARGET study ${ }^{1,2}$ of secondline sorafenib were reviewed. Patients in the sorafenib arm, compared with those in the placebo arm, had more hypertension, diarrhea and hand-foot skin reactions. However, patients in the placebo arm had a significant number of other side effects, including fatigue, tumour pain, bone pain and shortness of breath. The hand-foot skin reaction is the dose-limiting toxicity for sorafenib. ${ }^{1,2}$

\section{VEGF tyrosine-kinase inhibitors improve survival}

Although longer-term follow-up is still required, the TARGET study update ${ }^{2}$ presented at the ASCO 2006 meeting, showed that overall survival was improved before and after the crossover in patients receiving sorafenib, compared with those receiving placebo. For the first-line sunitinib study ${ }^{4}$ presented at the ASCO 2006 meeting, an overall survival curve was presented, but data were too preliminary to comment on.

\section{Other agents are active in $\mathrm{RCC}$}

Temsirolimus (CCI-779) is a drug with a different mechanism of action. It targets the mammalian target of rapamycin (mTOR), which is a downstream mediator of P13K/AKT, and thus, has a key role in controlling cellular division and growth. ${ }^{12}$ Results of the first-line study ${ }^{5}$ of temsirolimus were presented at the ASCO 2006 meeting. The study 
showed an improvement in progression-free survival (3.7 mo v. $1.9 \mathrm{mo} ; p=0.0001)$ and overall survival (10.9 mo v. $7.3 \mathrm{mo} ; p=0.0069)$ for poorrisk patients randomized to receive temsirolimus compared with those receiving interferon.

\section{What is not known about RCC?}

Unanswered questions about the optimal management of RCC remain.

\section{Should VEGF tyrosine-kinase inhibitors replace cytokines in the front-line?}

In Motzer's plenary discussion at the ASCO 2006 meeting, he said that "Sunitinib was the new reference standard for the first-line treatment of metastatic renal cell carcinoma and that this mechanism-directed renal cell carcinoma therapy based on tumor-specific molecular features is validated in kidney cancer and that Sunitinib is the new treatment option providing hope for patients with kidney cancer." However none of these drugs induce complete responses, unlike IL-2 and, very occasionally, interferon, in a small subset of patients. Bevacizumab and sorafenib are still being evaluated as first-line therapies. More data will emerge in the coming year to help answer the question about whether these targeted therapies will replace cytokines and which targeted therapy is superior.

\section{Should VEGF tyrosine-kinase inhibitors be given as neoadjuvant treatment before nephrectomy?}

The use of VEGF tyrosine-kinase inhibitors as neoadjuvant treatment before nephrectomy has not yet been studied. These targeted therapies can shrink primary cancers and may actually make surgery easier, but the optimal timing of the use of nephrectomy in conjunction with targeted therapy is not known.

\section{Should VEGF tyrosine-kinase inhibitors be given as adjuvant treatment?}

Studies are underway to answer the question about whether VEGF tyrosine-kinase inhibitors should be given as adjuvant treatment. However, results of these studies will take several years to become mature.

\section{How should we improve the efficacy of tyrosine-kinase inhibitors?}

To improve the efficacy of tyrosine-kinase inhibitors, we must ask ourselves questions about what the best schedule for these drugs is. For sunitinib, a continuous schedule, as opposed to 4 weeks on and 2 weeks off, may be more optimal. Vertical inhibition of the VEGF pathway in more than one way so that sunitinib or sorafenib could be added to an agent like bevacizumab, a monoclonal antibody to VEGF, may be more efficacious. Horizontal inhibition with sunitinib or sorafenib and a drug that targets a different mechanism of action, such as an mTOR inhibitor or immunotherapy, may provide more benefit. These concepts are currently being studied in phase 1 and 2 studies.

\section{Which is the best tyrosine-kinase inhibitor?}

At the plenary session of the ASCO 2006 meeting, Atkins outlined a possible algorithm for patients with metastatic RCC, based on phase 3 data, and suggested sunitinib or high-dose IL-2 as first-line therapy for good- or intermediate-risk patients, and temsirolimus for poor-risk patients. Sorafenib may be the best choice of second-line therapy for patients for whom treatment with cytokines failed. No data are available for those whose treatment with VEGF or mTOR inhibitor failed. However, a lack of phase 3 data for a particular agent and patient population does not mean that the drug lacks efficacy.

\section{Conclusion}

Treatment for metastatic RCC is moving from cytokines to targeted therapy. To date, 4 drugs have shown significant activity: sorafenib, sunitinib, bevacizumab and temsirolimus. Based on data presented at the ASCO meeting in June 2006, sunitinib will be the standard first-line therapy. Sorafenib used in second-line therapy improves progressionfree and overall survival, and temsirolimus prolongs overall survival in poor-risk patients.

\section{Practical experience with targeted therapy}

"Practical Experience with Targeted Therapy," presented by Dr. Lori Wood, a medical oncologist and associate professor at Dalhousie University, 
was designed to promote discussion about the practical aspects of using oral VEGF receptor tyrosinekinase inhibitors (sunitinib and sorafenib) in the clinic, and their toxicity and clinical responses. Most of the discussion was based on real-life experiences in the clinic, rather than on evidence from published literature, given the paucity of published literature by June 2006. Meeting participants had a wide range of experience with these agents, from significant to no experience.

\section{Toxicities}

Some of the toxicities of sunitinib and sorafenib are related to this class of drug, but specific toxicities are also seen with these 2 oral agents. Most oncologists think that all patients have some degree of toxicity, but the overall tolerability of these agents is better than that for immunotherapy. A range of different toxicities may necessitate dose reduction; often a dose is reduced because of the cumulative effects of a number of grade 2 toxicities instead of 1 or 2 grade 3 or 4 toxicities.

\section{Sunitinib}

The main symptoms that have necessitated a reduction in the dose of sunitinib are skin toxicity, fatigue, mucositis and gastrointestinal symptoms. Different types of skin toxicity can develop, but the particularly troubling type is a rash on pressure-bearing areas (e.g., the heels of the feet, the medial aspect of the metatarsophalangeal joint). This rash can be painful and interfere with daily functioning, such as walking and opening jars. Erythema is also seen on the soles of the feet and the palms of the hands, and around the nail beds. Patients often describe their skin as tight. Fatigue tends to be chronic, although it can improve during the 2-week break in the treatment regimen. Mucositis tends to be grade 1 or 2 , but can be grade 3 , and is often described as a burning sensation, or sensitivity to toothpaste or mouthwash and certain foods or temperatures. During examination, the mucosa often looks normal, compared with the appearance of 5-fluorouracil mucositis. Mucositis can also occur on the mucosa of the vagina, anus and urethra, causing significant discomfort. Gastrointestinal symptoms can vary, ranging from a very metallic taste in the mouth to dysgeusia, excess gas, nausea, or loose bowel movements to outright explosive, frequent bowel movements.

Patients usually feel well during the first 2 weeks of therapy. The toxicities, which tend to start toward the end of the second, or the start of the third or fourth week of therapy, may stay the same or progress. The symptoms tend to resolve quite quickly after stopping sunitinib, usually within 2 to 5 days.

In clinical trials with sunitinib, blood work has been done during cycle 1 on days 1, 14 and 28, and then on days 1 and 28 of each subsequent cycle. This frequency gives a good indication of any changes that may be occurring in the complete blood count and liver-function tests. Results of the white blood count and platelet count tend to be lowest during the blood work check done on day 28 of cycle 1 , at which time the physician may reduce the dose, as appropriate. If counts are low on day 14 of cycle 1 , the drug may have to be stopped early. Dose reduction for myelosuppression is often required in the first cycle, and unlike chemotherapy, for patients who develop neutropenia, it does not seem to be cumulative. Often patients will have a stable neutrophil count between 1.0 and 1.5 throughout several cycles of therapy. Thyroid function should be checked at regular intervals, and particularly in anyone suspected of having clinical hypothyroidism. Hypophosphatemia can also occur, so phosphorus levels should be checked on a regular basis.

Sunitinib may cause other reactions such as whitening of the hair, including eyebrows and moustaches; splinter hemorrhages; and yellowing of the skin while on the drug.

\section{Sorafenib}

Sorafenib also has a number of toxicities, which were reported during the abstract presentation at the ASCO 2005 and 2006 meetings. ${ }^{1,2}$ The doselimiting toxicity of sorafenib is skin toxicity, which occurs primarily in 2 different forms. One is the rash seen with hand-foot syndrome, which is similar to that seen with capecitabine. This rash occurs on the palms and the soles of the feet, especially in hyperkeratotic areas such as the heels or callused areas. The other rash can be a diffuse erythematous rash on the trunk, neck and face. Both of these rashes can simply be present, pruritic or painful, or can interfere with function. The key 
is to hold the drug before the rash becomes painful or interferes with function. If the drug is held until resolution starts (3-7 d), the drug may be reintroduced at the same dose, and the subsequent rash is often never as severe as the first and may even resolve over time. In other cases, however, the dose must be reduced, especially if the rash interferes with function.

Other side effects may also occur with sorafenib, such as fatigue and diarrhea. Diarrhea can range from loose stools to frank explosive diarrhea. Anecdotally, cholestyramine and other agents traditionally used with chemotherapy, such as loperamide (Imodium), have been reported to improve these side effects.

During the first 4-6 weeks of therapy, the patient's blood pressure must be monitored since it is typically during this period that hypertension can develop. Patients' pretherapy blood pressure must be documented, and patients should be given specific guidelines about when to contact the clinic in case adjustments in their antihypertensive drugs must be made or the antihypertensive drugs must be added.

\section{Drug interactions}

Both sorafenib and sunitinib have a number of potential drug interactions that need to be continuously reviewed if patients are prescribed new drugs, or take any over-the-counter or herbal remedies.

\section{Clinical observations}

The length of time for patients to respond to therapy with sorafenib and sunitinib can be quite variable. For some patients, visible regression (e.g., of skin metastases) or symptomatic improvement can be seen within a few days; for others, radiographic response occurs over several months. Mixed responses seem much more common with targeted therapy than with immunotherapy. A key message delivered at the meeting was not to discontinue therapy too soon, provided the patient's tolerance is satisfactory. In one case cited, a patient who had a true partial response in the lung and mediastinal lymph nodes after 3 cycles of therapy developed new adrenal metastases. However, because of continued clinical benefit derived from the therapy, the patient continued on the clinical trial and after 4 further cycles of therapy, the new adrenal metastases started to regress.

Clinical and radiographic observations about the schedule of therapy with sunitinib (4 weeks on and 2 weeks off) were discussed. Some patients seem to have clinical or radiographic progression during their 2-week rest period (e.g., worsening skin metastases, pleural effusions and ascites). Some of these patients had the opportunity to switch to a continuous dosing schedule that involved 4 weeks on continuous drug, starting at $37.5 \mathrm{mg}$, and resulted in stabilization or improvement in their disease status. As a consequence, radiographic evaluation should be done near day 28 of the 6-week cycle instead of at the end of the 2-week break to ensure correct interpretation of drug activity.

Anecdotally, responses to targeted therapy have been reported for papillary renal cell cancer and RCC with sarcomatoid differentiation (SD). However, whether the anticancer activity is as good as that for clear-cell histology is unknown. Individual medical oncologists treating patients with brain metastases have seen some central nervous system responses.

The consensus of the group who had experience with the targeted agents is that they definitely have clinical benefit and the ability to shrink tumours in patients with RCC, although complete responses are rare. These agents are tolerable; however, just like any anticancer therapy, they have bothersome and potentially life-threatening side effects that must be managed by an experienced team of physicians and nurses.

\section{Sarcomatoid renal cell carcinoma}

The last discussion during the Renal Cell Cancer Forum, titled "Sarcomatoid Renal Cell Carcinoma," was presented by Dr. Neil Reaume, a medical oncologist and assistant professor in the Department of Oncology at the Ottawa Regional Cancer Centre. The emphasis of this talk was on whether patients with sarcomatoid RCC should be treated differently. At the outset of the discussion, participants were reminded that the literature on this topic is scant, made up predominantly of case reports and case series, all of which are retrospective. Two different cases were discussed to illustrate some of the key points about sarcomatoid RCC. 


\section{Background}

SD, which can occur in any type RCC histology subtype, presents as a transformation into a highgrade tumour with a spindle-cell appearance and immunohistochemical evidence of epithelial and mesenchymal components. ${ }^{13}$ In the past, this type of tumour has also been called carcinosarcoma, metaplastic carcinoma or spindle-cell carcinoma. Morphologic patterns of SD include fibrosarcomalike, malignant fibrous histiocytoma-like, rhabdomyosarcomatous and undifferentiated tumours. SD was recognized formally by the joint Union Internationale Contre le Cancer and the American Joint Committee on Cancer working group in 1997.

The largest series, reported in 2001 by de PeraltaVenturina and colleagues, ${ }^{14}$ included 101 cases of RCC with SD from a retrospective review of all RCC cases from 4 centres, 2 in the United States and 2 in Switzerland. Of the US cases, 74 of 952 consecutive RCC patients had SD, for an overall incidence of $8 \%$. By subtype, SD was associated with $8 \%$ of clear-cell carcinomas, and with $3 \%$ of papillary, $9 \%$ of chromophobe, $29 \%$ of collecting-duct and $11 \%$ of unclassified tumours. However, these numbers are highly variable because of the small number of cases. RCC with SD tended to be diagnosed at a more advanced stage. The overall survival was also worse for those RCC patients with SD than those without; 5-year and 10-year survival for patients with SD was $22 \%$ and $13 \%$, respectively, as opposed to $79 \%$ and $76 \%$, respectively, for those without $(p=0.0001)$. Those tumours with less than $50 \%$ SD had a better outcome than those that had more than $50 \%(p=0.0453)$. This is also true if lymphovascular invasion was present. When a Cox proportional hazard regression model was run, the most significant variable was the stage of the cancer: those patients with stage III and IV RCC did worse than those with stage II. The median survival of 19 months for patients in this study was significantly longer than that of the 4 other case series, ${ }^{15-18}$ published between 1968 and 1987, that involved 13-42 patients who had RCC with SD (range 6-12 mo survival).

\section{Treatment}

Different treatment modalities, namely, immunotherapy, chemotherapy and targeted therapy, were discussed. In clinical trials, patients with non-clear cell pathology make up less than $10 \%$ of those enrolled in clinical trials, and the amount of SD is rarely reported in RCC trials. Some studies also limit participation to patients with clear-cell RCC only. In 2 case series ${ }^{19,20}$ of non-clear cell pathology, 64 and 17 cases treated with immunotherapy demonstrated very poor response rates (less than $6 \%$ ). A third case series report, ${ }^{21}$ a retrospective review of 31 patients who had RCC with SD, 24 of whom had metastatic disease, focused on response to combined surgical resection and immunotherapy. Eighty-one percent of patients received immunotherapy with low- or high-dose IL-2, vaccine or interferon. In this patient population, 2 had complete responses and 5 had partial responses. The patients who had high-dose IL-2 seemed to have better survival than those who did not receive high-dose IL-2 therapy $(p=0.0175)$.

The majority of the literature dealing with chemotherapy is made up of case reports of 1-4 patients or case series. Doxorubicin-based chemotherapy is most commonly reported. Discussion focused on 2 studies in particular. In the study of Escudier and colleagues, ${ }^{22} 23$ patients were treated with doxorubicin and ifosfamide; none responded, but 6 patients had stable disease. In the second, a single-arm, single-institution study of 18 patients by Nanus and colleagues, ${ }^{23} 10$ patients had RCC with SD and the others had rapidly progressing RCC. Patients were given doxorubicin and gemcitabine every 2-3 weeks (median 5 cycles given) with granulocyte colony-stimulating factor. There were 2 complete responses and 5 partial responses; the median duration of response was 5 months. The 2 complete responses were in patients who had SD. No grade 4 toxicities occurred. Results of this study have led to an ongoing prospective multicentre phase 2 Eastern Cooperative Oncology Group study of this regimen.

As of June 2006, little published evidence exists on which to judge whether targeted therapies work. Anecdotally, however, oncologists at the conference indicated that they had seen responses with these agents in patients with SD. The question remains about whether they are better than chemotherapy or immunotherapy and whether the responses are durable.

\section{Conclusion}

In summary, SD can be seen with any histologi- 
cal subtype of RCC, with any grade or stage, and RCC with SD tends to occur at a more advanced stage. Adverse prognostic factors are greater than $50 \% \mathrm{SD}$, vascular invasion and more advanced stage. Different systemic therapies may offer varying benefits to individual patients; however, based on the evidence, it is impossible to identify the optimal therapy. This patient population is underrepresented and underreported in clinical trials.

\section{Summary}

Overall, the First Annual Genitourinary Medical Oncology Conference was a great success. The conference provided an opportunity to discuss the management of both RCC and testicular cancer. The exciting advancements in the management of RCC will continue to lead to further successes and challenges in the future.

From the *Division of Medical Oncology, Queen Elizabeth II Health Sciences Centre, Halifax, NS, the †Institut Gustave Roussy, Villejuif, France, and the †OHtawa Regional Cancer Centre, Ottawa, Ont.

This article has been peer reviewed.

Competing interests: None declared for Dr. Wood. Dr. Rheaume has received speaker fees, educational grants or travel assistance from Pfizer, Bayer, Wyeth, and Lilly. Dr. Escudier has received honoraria from Bayer Healthcare, Roche and Wyeth Pharmaceuticals, Novartis, and Inate Pharma.

\section{References}

1. Escudier B, Szczylik C, Eisen T, et al. Randomized phase III trial of the Raf kinase and VEGFR inhibitor sorafenib (BAY 43-9006) in patients with advanced renal cell carcinoma (RCC) [abstract LBA4510]. 2005 ASCO Annual Meeting Proceedings; May 13-17, 2005; Orlando (FL): Database Publishing Group; 2005. p. 380s.

2. Eisen $T$, Bukowski RM, Staehler M, et al. Randomized phase III trial of sorafenib in advanced renal cell carcinoma (RCC). [abstract 4524] 2006 ASCO Annual Meeting Proceedings; June 2-6, 2006; Atlanta (GA): Database Publishing Group; 2006. p. 223s.

3. Motzer RJ, Rinni BI, Michaelson MD, et al. Phase 2 trials of SU1 1248 show antitumor activity in second-line therapy for patients with metastatic renal cell carcinoma (RCC) [abstract 4508]. 2005 ASCO Annual Meeting Proceedings; May 13-17, 2005; Orlando (FL): Database Publishing Group; 2005. p. 380s.

4. Motzer RJ, Hutson TE, Tomczak P, et al. Phase III randomized trial of sunitinib malate (SU1 1248) versus interferon-alfa (IFN- $\propto$ ) as first-line systemic therapy for patients with metastatic renal cell carcinoma (mRCC) [abstract LBA3]. 2006 ASCO Annual Meeting Proceedings; June 2-6, 2006; Atlanta (GA): Database Publishing Group; 2006. p. 2s.

5. Hudes $G$, Carducci $M$, Tomczak $P$, et al. A phase III randomized, 3-arm study of temsirolimus (TEMSR) or interferon-alpha (IFN) or the combination of TEMSR + IFN in the treatment of first-line, poor-prognosis patients with advanced renal cell carcinoma [abstract LBA4]. 2006 ASCO Annual Meeting Proceedings; June 2-6, 2006; Atlanta, Georgia: Database Publishing Group, Inc.; 2006. p. 2s.

6. Escudier B, Eisen T, Stadler WM, et al. Sorafenib in advanced clear-cell renal-cell carcinoma. N Engl J Med 2007;356:125-34.

7. Motzer RJ, Hutson TE, Tomczak P, et al. Sunitinib versus interferon alfa in metastatic renal-cell carcinoma. N Engl J Med 2007;356:115-24

8. Medical Research Council Renal Cell Collaborators. Medical Research Council (MRC) Renal Cell Collaboration. Interferon- $\propto$ and survival in metastatic renal carcinoma: early results of a randomized controlled trial. Lancet 1999;353:14-7.

9. Negrier $S$, Perol D, Ravaud A, et al. Do cytokines improve survival in patients with metastatic renal cell carcinoma (MRCC) of intermediate prognosis? Results of the prospective randomized PERCY Quattro trial [abstract LBA451 1]. 2005 ASCO Annual Meeting Proceedings; May 13-17, 2005; Orlando (FL): Database Publishing Group; 2005. p. 380s.

10. Negrier $S$, Escudier $B$, Lasset $C$, et al. Recombinant human interleukin-2, recombinant human interferon alfa-2a, or both in metastatic renal-cell carcinoma. N Engl J Med 1998;338:1272-8

11. Lamuraglia $M$, Chami L, Escudier $B$, et al. Doppler ultrasonography with perfusion software and contrast agent injection as an early evaluation tool of metastatic renal cancers treated with RAF kinase and VEGFR inhibitor: a prospective study [abstract 803]. 2005 European Cancer Conference (ECCO) 13; October 30-November 3, 2005; Paris, France: Elsevier; 2005. p. 229.

12. Atkins MB, Hidalgo M, Stadler WM, et al. Randomized phase II study of multiple doses levels of $\mathrm{CC}-779$, a novel mammalian target of rapamycin kinase inhibitor, in patients with advanced refractory renal cell carcinoma. J Clin Oncol 2004;22:909-18.

13. Storkel S, Eble JN, Adlakha K, et al. Classification of renal cell carcinoma: Workgroup No. 1. Union Internationale Contre le Cancer (UICC) and the American Joint Committee on Cancer (AJCC). Cancer 1997;80:987-9.

14. de Peralta-Venturina M, Moch H, Amin M, et al. Sarcomatoid differentiation in renal cell carcinoma: a study of 101 cases. Am I Surg Pathol 2001;25:275-84.

15. Bertoni F, Ferri C, Benati A, et al. Sarcomatoid carcinoma of the kidney. J Urol 1987; 137:25-8.

16. Ro JY, Ayala AG, Sella A, et al. Sarcomatoid renal cell carcinoma: clinicopathologic A study of 42 cases. Cancer 1987;59:516-26.

17. Tomera KM, Farrow GM, Lieber MM. Sarcomatoid renal carcinoma. J Urol 1983; 130:657-9

18. Farrow GM, Harrison EG Jr, Utz DC. Sarcomas and sarcomatoid and mixed malignant tumors of the kidney in adults - III. Cancer 1968;22:556-63.

19. Motzer RJ. Treatment outcome and survival associated with metastatic renal cell carcinoma of non-clear-cell histology. J Clin Oncol 2002;20:2376-81.

20. Upton MP, Parker RA, Youmans A, et al. Histologic predictors of renal cell carcinoma response to interleukin-2-based therapy. J Immunother 2005;28:488-95.

21. Cangiano T, Liao J, Naitoh J, et al. Sarcomatoid renal cell carcinoma: biologic behavior, prognosis, and response to combined surgical resection and immunotherapy. J Clin Oncol 1999;17:523-8

22. Escudier B, Droz JP, Rolland F, et al. Doxorubicin and fosfamide in patients with metastatic sarcomatoid renal cell carcinoma: a phase II study of the Genitourinary Group of the French Federation of Cancer Centers. J Urol 2002;168:959-61.

23. Nanus DM, Garino A, Milowsky MI, et al. Active chemotherapy for sarcomatoid and rapidly progressing renal cell carcinoma. Cancer 2004;101:1545-51.

Correspondence: Dr. Lori Wood, Division of Medical Oncology, QEEll Health Sciences Centre, Rm. 471, 1278 Tower Rd., Halifax, NS B3H 2Y9; Lori.Wood@cdha.nshealth.ca 